\title{
Motion and color generate coactivation at postgrouping identification stages
}

\author{
Leo Poom
}

Published online: 30 April 2011

(C) Psychonomic Society, Inc. 2011

\begin{abstract}
Response times (RTs) were measured in a postgrouping visual identification task. Shapes composed of multiple elements were distinguished by color, motion, orientation, and spatial frequency alone or in pairwise conjunctions. The largest amount of redundancy gain, requiring coactivation as revealed by a race model analysis, was obtained with color-motion conjunctions. In contrast, RTs for a pregrouping detection task using the same target shape as in the identification task, distinguished by color, motion, or a conjunction of these features, showed no evidence for coactivation. The results provide psychophysical evidence for coactivation of color and motion signals in cortical regions specialized for grouping and object identification, as opposed to separate processing of these features in cortical area V1, believed to limit performance in visual search and pregrouping detection.
\end{abstract}

Keywords Race model $\cdot$ Identification $\cdot$ Feature integration $\cdot$ Response time $\cdot$ Grouping

Objects can be distinguished and identified visually by contrasts in any of several features. For example, the same object can be identified whether it has a different color, brightness, or texture than the surrounding scene, and when camouflaged against a textured background, it immediately reveals itself when moving and can be identified by relative motion alone. Identification is possible even when constituent parts of an object are spatially separated, requiring grouping, and the parts are distinguished by different features (Poom,

L. Poom $(\bowtie)$

Department of Psychology, Uppsala University,

Box 1225, SE-751 41, Uppsala, Sweden

e-mail: Leo.Poom@psyk.uu.se
2001, 2002). Although it has been debated to what extent features are segregated in feature-specific channels, results providing evidence for segregation of color and motion have been used as a cardinal example to argue in favor of separate feature-specific processing. For example, color and motion are, to a high degree, segregated at the first stages of visual processing involved in visual search tasks, where odd targets embedded in distractors have to be detected, whereas other feature conjunctions produce coactivation (Koene \& Zhaoping, 2007; Poom, 2009). Here, coactivation refers to summing of activity, or saliency, on a common neural population.

It has long been known that response times (RTs) are shorter in redundant conditions when a target is specified by two constituent parts than if a single part is used (Miller, 1982; Raab, 1962; Todd, 1912). Also, in simple visual search tasks, where a target is embedded in distractor elements, redundancy gain typically occurs when two features distinguish a target - for example, orientation and spatial frequency (Koene \& Zhaoping, 2007; Poom, 2009). It is possible to use experimental psychological methods to investigate whether the output from the decision stage can be accounted for by separate feature-specific processing or whether it relies on coactivation. If decisions are based on activities in separate channels and their time-to-reach-threshold distributions overlap, a statistical redundancy gain is possible, as formulated by the race model. On average, then, the time for the winner to reach criterion is less than the time for either channel alone (Miller, 1982; Raab, 1962). The race model sets an upper limit on the redundancy gain. Coactivation models, on the other hand, may produce redundancy gain above the gain predicted by two separate channels (e.g., Miller, 1982; Mordkoff \& Yantis, 1993).

RT analysis and violations of the race model are frequently used as evidence for the influential saliency map models of visual search in which feature contrast 
signals are summed on a master saliency map, producing RTs that are shorter than would be predicted by separate activities (Zehetleitner, Krummenacher, \& Müller, 2009). The redundancy gain in a visual search task obtained for color-orientation and orientation-motion combinations (Koene \& Zhaoping, 2007), and spatial frequency combined with orientation, color, and motion, respectively (Poom, 2009), is too large to be explained by statistical facilitation from separate channels and requires coactivation. Neither study, however, showed any evidence of coactivation for color-motion combinations. In line with a saliency map summation model where feature contrasts on the same location add activity on the same neural population, coactivation was location specific, since it was found only when both features distinguished a single target, and not separate targets (Poom, 2009).

Whereas the above-mentioned detection tasks rely on pregrouping stages, in postgrouping tasks, multiple elements are grouped into a larger shape. Field, Hayes, and Hess (1993) used a set of oriented Gabor elements that created a contour to investigate postgrouping stages, and Nothdurft (1993) demonstrated that grouping by common motion and common color are two strong grouping principles. In the above-mentioned studies, grouping principles were investigated using detection tasks primarily driven by bottom-up processes. It is largely unknown, however, how features combine in a postgrouping identification task involving top-down processes believed to occur hierarchically subsequent to processes crucial for detection and visual search tasks.

In Experiments 1 and 2, RTs were measured for identification of a well-known target (an S-shape) composed of multiple elements, each distinguished by a single feature or two features in conjunction. In Experiment 2, a detection task was included. In brief, the results showed that, contrary to detection, color-motion conjunctions in the identification task produced a redundancy gain too large to be accounted for by separate processing. Furthermore, the redundancy gain for identification was larger for the color-motion conjunction than for any other feature conjunctions tested.

\section{The race model}

Suppose that two feature-specific channels are sensitive to features $f_{A}$ and $f_{B}$ respectively, and $f_{A \& B}$ indicates that both features are present. Then the race model inequality originally formulated by Miller (1982) can be written as

$P\left(R T<t \mid f_{A \& B}\right) \leq P\left(R T<t \mid f_{A}\right)+P\left(R T<t \mid f_{B}\right)$.

The left side of the inequality is the empirically obtained cumulative distribution functions (CDFs) of RTs less than $t$ from redundant-feature conditions. The right side is the sum of corresponding CDFs from single-feature conditions. That is, cumulative distributions for RTs at each quantile (i.e., points taken at regular intervals from the $\mathrm{CDF}$ ) when two features are combined never reaches beyond the sum obtained from conditions where each feature is used alone.

The race model embraces several models with varying degrees of channel independence, including those where the activities of separate channels are correlated and those where times to reach criterion are independent between channels, and models where the time to reach criterion for each channel is independent of stimulation levels of the other channel (Hughes \& Townsend, 1998). If the race model inequality is violated at any quantile, race models must be rejected in favor of coactivation models. Coactivation, however, cannot be rejected if the race model boundary is not violated. Equivalently, inequality 1 may be expressed as the minimum RT allowed at each percentile, which then sets a race model RT boundary; evidence for coactivation occurs when RTs are shorter than this boundary.

In neural coactivation models, it is assumed that separate features produce activity in a common channel before the decision stage (Miller, 1982). This coactivation may lead to boosted activity that satisfies the criterion faster than a statistical redundancy gain as expressed by inequality 1 .

There is a possibility that redundancy gain, measured as RTs, may occur at the motor level, with faster motor activity using redundant targets (Diedrich \& Colonius, 1987), but investigations addressing this issue suggest that this is not the case (Mordcoff, Miller, \& Roch, 1996), although other post perceptual processes cannot be excluded (Feintuch \& Cohen, 2002).

\section{Experiment 1}

Postgrouping identification RTs were measured with shapes distinguished by color, motion, orientation, or spatial frequency (single-feature conditions) or by two of these features in conjunction (double-feature conditions). RTs were measured for identifying an " $\mathrm{S}$ " target shape composed of 11 elements embedded in 89 distractor elements on $75 \%$ of the trials. On the remaining trials, 1 of 11 nontarget shapes was randomly presented, also composed of 11 elements, and the participants were asked to withhold their response (Fig. 1a). An example of displays with the S-shaped target distinguished by orientation alone is shown in Fig. 1b, by spatial frequency alone in Fig. 1c, and by orientation and spatial frequency in conjunction in Fig. 1d. The features, display, stimulus parameters, apparatus, design, and procedures are the same as those used in the detection study (Poom, 2009). 
Fig. 1 Stimuli. a Schematic view of the 12 shapes composed of 11 elements used in the experiment. The S-shaped target is enclosed. In the real display, shape elements were distinguished from distractor elements by color, orientation, spatial frequency, and motion, either alone or in pairwise conjunctions. Examples of stimulus displays are shown with the target S-shapes distinguished by b orientation and $\mathbf{c}$ spatial frequency in single-feature conditions and by $\mathbf{d}$ spatial frequency and orientation in conjunction in a double-feature display

A
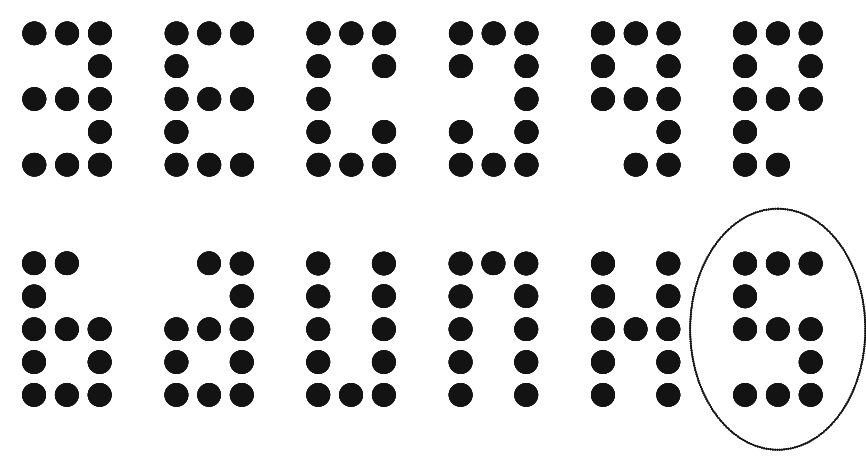

B

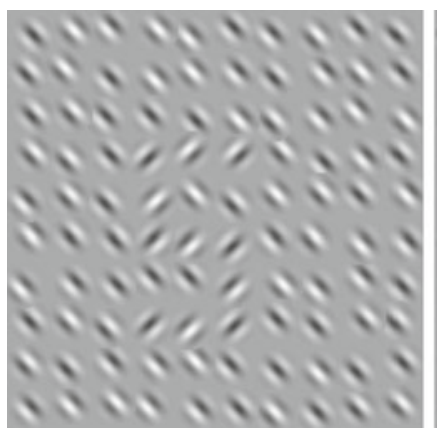

C

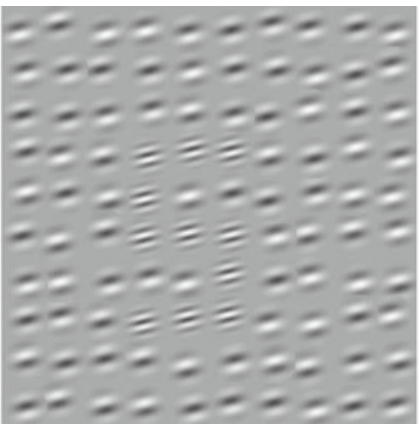

D

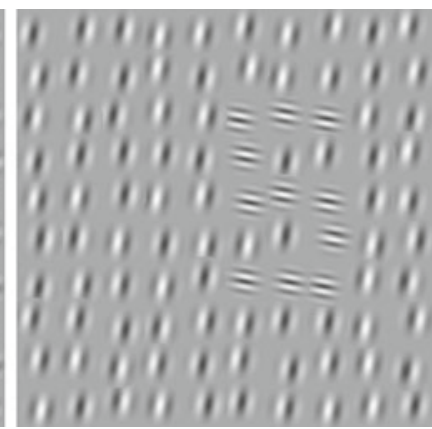

\section{Method}

Participants Nineteen participants (19-45 years old) with no color vision deficits were recruited to participate in the experiment. All had normal or corrected-to-normal vision. Informed consent was obtained from all the participants after the nature of the experiment was explained, and they were rewarded with cinema tickets for their participation.

Apparatus and stimuli Stimuli were displayed on a 17-in. $1,280 \times 960$ pixel resolution CRT monitor with 24-bit color resolution and a $100-\mathrm{Hz}$ refresh rate run by a $\mathrm{PC}$ equipped with an Intel Pentium 4 processor. The mouse track ball was removed to increase the accuracy of timing (Chambers \& Brown, 2003; Segalowitz \& Graves, 1990).

The stimulus area occupied $13^{\circ} \times 13^{\circ}$ of visual angle from a viewpoint $60 \mathrm{~cm}$ from the screen and consisted of a regular array of 10 rows and 10 columns of cells. Each cell was occupied by a Gabor element randomly scattered up to $0.25^{\circ}$ around the cell midpoint. The brightness of each element, appearing with about $0.8^{\circ}$ diameter, was modulated by a Gabor function (a spatial two-dimensional Gaussian blob, with size $\sigma=0.22^{\circ}$, multiplied with a sine function) according to the equation $L=L o+c \cdot \mathrm{G}(x, y, a, f)$, where $L o$ is background luminance set by the RGB values, $c$ is luminance contrast, $\mathrm{G}(x, y, a, f)$ is the Gabor function, $x$ and $y$ are spatial positions, $a$ is the element orientation, and $f$ is spatial frequency. The luminance contrasts of all shape and background elements were assigned random values. The
Michelson contrast had a rectangular distribution between $0.38\left(I \max =45, I \min =20 \mathrm{~cd} / \mathrm{m}^{2}\right)$ and $0.72(I \max =60$, $I \min =10 \mathrm{~cd} / \mathrm{m}^{2}$ ) against the $30-\mathrm{cd} / \mathrm{m}^{2}$ background with $L o$ set to 60 . This contrast noise minimized any possible luminance-based saliency artifacts when color was used to distinguish shape elements. The element spatial frequency was 1.5 cycles/deg, corresponding to a spatial period of $0.67^{\circ}$. A common randomly determined orientation was given to all the elements on each trial, but each element was given a random spatial phase. Shapes were presented at randomly selected locations at the inner $8 \times 8$ array of cells.

All shapes were composed of 11 elements distinguished from the background elements by motion, orientation, spatial frequency, or color used in isolation or pairwise combined. Except when shapes were distinguished by color, all the elements were achromatic. These shape elements were located at selected positions of 13 elements arranged in an " 8 " figure within an area three columns wide and five columns high. A total of 12 shapes were used in the experiment, and the target presented on go trials was an "S" figure, whereas the other shapes presented on no-go trials were distractor shapes (Fig. 1a). Shapes were defined either by one feature in isolation (single-feature conditions; Figs. 1b, c) or by two features (double-feature conditions; Fig. 1d).

When color was used to distinguish the shape elements, the color saturation, expressed in RGB values, was spatially modulated between background gray and purple-red in phase with the luminance, as stated by 
the following equations $\mathrm{R}=L / 3+S \cdot \operatorname{Abs}[\mathrm{G}(x, y, a, f)]$; $\mathrm{G}=L / 3-S \cdot \operatorname{Abs}[\mathrm{G}(x, y, a, f)] ; \mathrm{B}=L / 3$, where $S$ is max color saturation (set to 50, and $L o$ was set to 60); consequently, $\mathrm{R}+\mathrm{G}+\mathrm{B}=L$. As a result, the shape elements distinguished by color appeared with successive bright and dark purple-red stripes, while the background elements had bright and dark achromatic stripes. Possible luminance-based artifacts induced by the color contrast were efficiently camouflaged by the random contrast variation between all elements.

When motion was used to distinguish the target, the Gaussian envelope of each element was stationary, but the spatial frequency pattern was phase shifted $90^{\circ}$ between the frames; that is, the spatial frequency pattern was moved one fourth period between frames. Motion sequences consisted of four frames. Each frame was presented for $50 \mathrm{~ms}$ or five monitor frames. The sequence of four frames was repeated, creating the appearance of unidirectional motion with temporal frequency of 5 cycles/s, while the background elements were static. Although 50-ms motion frames were used, the spatial phase shift of the Gabor patterns between frames was small, resulting in the appearance of quite smooth motion. The first frame after initializing a trial was visible for only $20 \mathrm{~ms}$ to minimize RT delays.

Shape elements distinguished by spatial frequency had a frequency of 2.6 cycles/deg, corresponding to a spatial period of $0.38^{\circ}$ which was 0.56 times the period of the background elements (Fig. 1c). Shape elements distinguished by orientation were oriented orthogonally to the distractor elementsthat is, $90^{\circ}$ orientation contrast (Fig. 1b).

Procedure Different pairs of features were run in separate sessions. Six sessions were run with pairwise combinations of motion, color, spatial frequency, and orientation. Each session included three blocks: two single-feature blocks and one double-feature block where both features distinguished the shape. The three blocks were presented in random order within each session. On average, $25 \%$ of randomly scattered trials were no-go trials with distractor shapes. The blocked session procedure is equivalent to the procedure used in the detection task (Poom, 2009).

Before each session, participants performed 20 practice trials with the same features as those used in the session. The participants initiated each session by pressing the space bar. When the S-shaped target appeared, they responded by clicking the mouse left button with their right-hand index finger (go trial). If a distractor shape appeared, they were asked to withhold any response (no-go trial). After a response or after $1.5 \mathrm{~s}$ if no response was given, the stimulus array disappeared, and after 2.5-3 s, the next stimulus appeared. This was repeated until 15 successful go trials were collected in each block. Participants were asked to be as accurate and as fast as possible. After each trial, the number of correct trials within the session so far was displayed in a rectangular area in the center of the display, which turned green after correct trials and red after incorrect trials to provide feedback.

The number of trials varied in each session, since the $25 \%$ chance of no-go trials was independently assigned on each trial and every wrong response resulted in an extra trial. A total of six sessions, one for each possible feature combination, were run, with an average of about 60 trials each, resulting in a total of about 360 trials to complete one experiment for each participant. RTs shorter than $150 \mathrm{~ms}$ were considered as anticipations and were excluded from the analysis. Short breaks were allowed between sessions, and the experiment took about $40 \mathrm{~min}$ to complete.

Data analysis An algorithm developed by Ulrich, Miller, and Schrötter (2007), which does not require any large number of observations and allows the results to be aggregated across participants, was used to perform the race model evaluation. The algorithm estimates empirical CDFs for each participant and every stimulus condition and calculates the race model boundary $B$ by adding the corresponding single-feature CDFs (right side of inequality 1 ). The CDF for doublefeature condition $D$ is also calculated. The $D$ and $B$ RTs are then compared at 10 percentiles $(5 \%, 15 \%, 25 \%, \ldots 95 \%)$ for each participant. Race model violations occur if the difference $B-D$ is significantly positive, which is evaluated by computing a separate paired $t$-test at each percentile across participants, and type I errors are avoided by using Bonferroni corrections (Ulrich et al., 2007). The race model inequality is rejected, and coactivation must be accepted if there is a significant violation of the inequality at any percentile.

\section{Results}

Figure 2 shows the total mean RTs for each feature combination and corresponding single features. Overall, RT for identification in this task is about $500 \mathrm{~ms}$.

It can be noted from inequality 1 that race model violations during coactivation are bound to occur at lower cumulative probabilities, since the left side of the inequality asymptotes at 1 and the right side asymptotes at 2 . This is clearly revealed in Fig. 3, where the double-feature RT at each percentile is subtracted from the race model RT boundary (Poom, 2009). Race model violations are clearly indicated by positive values, and significant violations are marked with crosses.

Redundancy gain with the largest race model violations occurred for color-motion conjunctions, providing strong evidence for coactivation. These violations, evaluated separately at 10 percentiles across 19 participant, were 


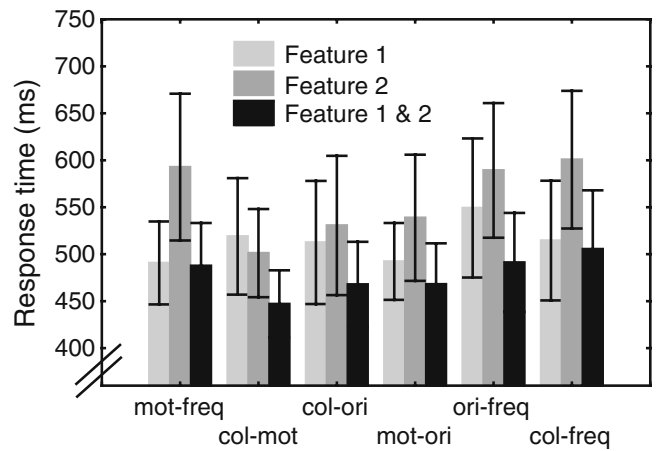

Fig. 2 Average response times (RTs) across participants are shown for single and double features for each feature combination. The light gray and dark gray bars show RTs for feature 1 and feature 2, respectively, in the same order as indicated on the abscissa. The whiskers show $95 \%$ confidence intervals

significant at 4 percentiles between the 5 th and 35 th percentiles, $t \mathrm{~s}(18)>2.5, p \mathrm{~s}<.05$. Motion-orientation produced significant violations at the 5 th and 15 th percentiles, $t \mathrm{~s}(18)>2.3, p \mathrm{~s}<.05$, and orientation-spatialfrequency produced a significant violation at the 15 th percentile only, $t(18)=2.1, p<.05$. The color-orientation, color-spatial-frequency, and motion-spatial-frequency conjunctions produced no statistically significant violations at any percentile, although, at the lowest percentiles, the race boundary appeared slightly trespassed.

False alarm (FA) rates varied between $19 \%$ for singlefeature conditions for color-defined shapes and $22 \%$ for orientation-defined shapes. Corresponding values for double-feature conditions were $20 \%$ for motion-orientation conjunctions and $22 \%$ for orientation-color conjunctions. For all conditions, the number of misses, failure to press the key on go trials, was below $1 \%$, with an average of $0.5 \%$. The number of anticipations (RT $<150 \mathrm{~ms}$ ) was very low.

\section{Discussion}

The most interesting result from Experiment 1 is that race model violation occurred with the color-motion conjunction, which is contrary to results obtained in a similar detection task (Poom, 2009). Decisions based on activities in separate channels can be excluded, and coactivation is demonstrated by the race model violations. Failure to find such violations, however, can be accounted for by statistical
Fig. 3 Response time (RT) deviations from the race model boundary (line at zero) from Experiment 1, separately presented at each percentile. The differences between the boundary and RTs from the double-feature conditions are displayed with filled disks, and significant violations (positive signs) are displayed with crosses

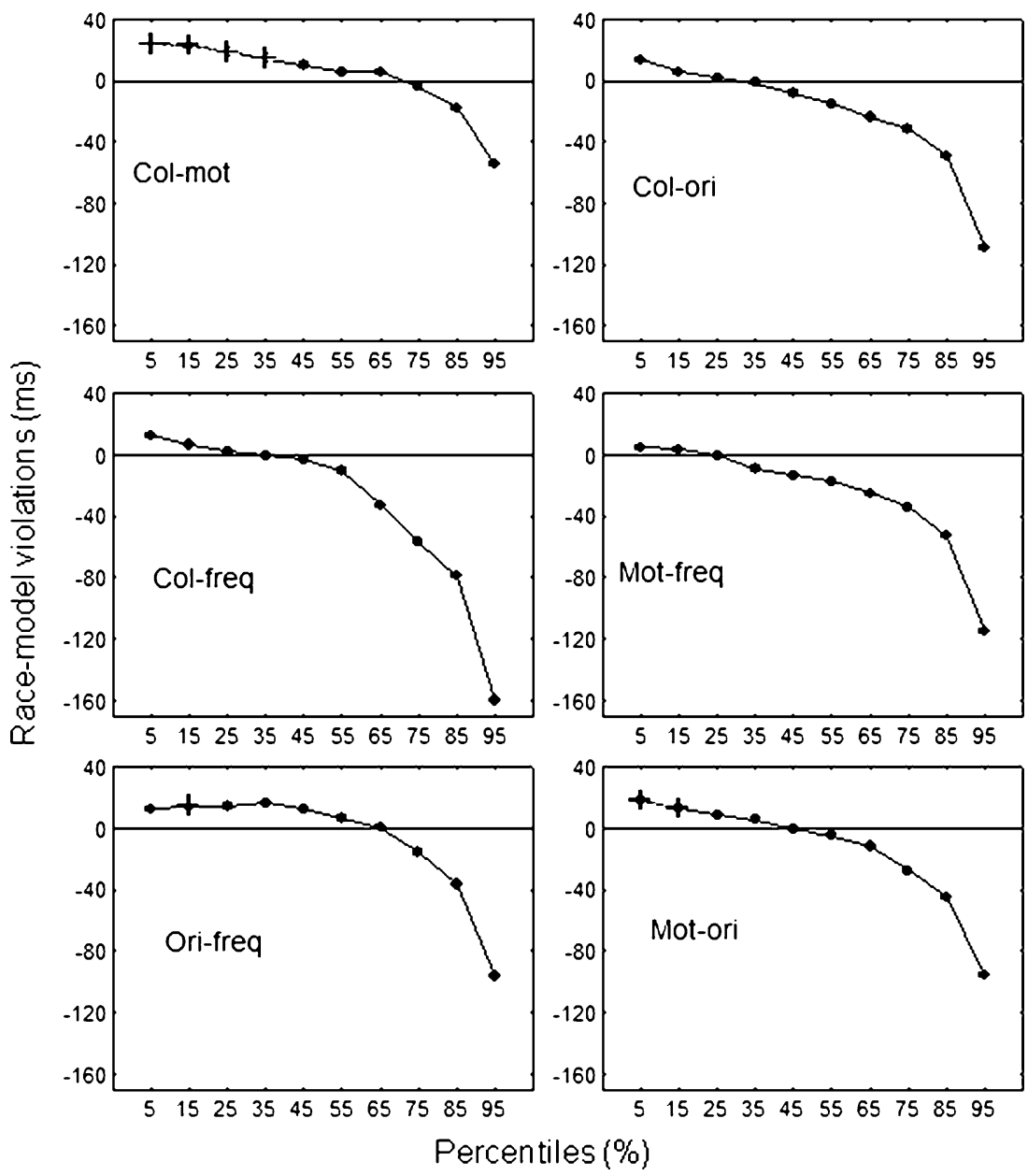


facilitation from separate activities. Besides color-motion conjunctions, the only feature combinations that gave significant violations of the race model were orientationmotion and orientation-spatial-frequency.

The detection of motion and orientation combined could result from differential velocity signals due to the orientation difference of the Gabor elements (von Mühlenen \& Müller, 2001). In their experiments, von Mühlenen and Müller showed that the detection of a moving target line, embedded in moving and stationary distractors of various orientations, was facilitated due to differential motion signals. The orientation difference of the target line changes the motion signal relative to the other elements. Here, all background elements were always stationary, so the motion difference was the same in both the motion-alone condition and the orientation-motion conjunction, although the orientation was the same in the first condition and orthogonal in the second condition. Perhaps the orientation contrast produced a greater relative motion signal, so the orientation difference may have caused a motion signal that was greater than when orientation was the same among all the elements. So, the shorter RTs could have been caused by a change in the motion signal itself, due to an orientation difference. Similarly, the shorter RT obtained with a combination of orientation and spatial frequency may have resulted from stronger orientation signals with higher spatial frequencies. This process is not due to coactivation in a strict sense, where separate features produce a common signal. Instead, this facilitation is caused by the increased saliency of a single feature. For example, rather than producing conjoint activity from spatial frequency and orientation, orientation saliency itself may increase with increases in the number of stripes signaling the orientation in the same way as lightness increases with increases in the number of turned-on light bulbs. I thank von Mühlenen for suggesting that, in some cases, the change in one feature may change the saliency of other features.

The presence, as compared with the absence, of violations of the race model inequality suggests qualitatively different structures of the processing architectures. Still, it has been proposed that the summed violations across percentiles, which is an estimate of the area between the zero level and positive race model violation curve, can be used as an index for comparing results between race model analyses (Colonius \& Diederich, 2006). In Fig. 4, this index for each feature combination is compared for the present identification data from Experiment 1 and the detection data obtained from the visual search task (Poom, 2009). Positive violations are summed across percentiles for each participant and then averaged across participants separately for each feature combination.

Inspection of Fig. 4 shows that for the color-motion combinations produced larger summed violations in the identification task of Experiment 1 than in the detection task. The opposite pattern was obtained for the motionorientation combinations as revealed by the nonoverlapping $95 \%$ confidence intervals. Orientation-frequency also shows quite a large opposite pattern, although confidence intervals overlap.

Double-feature conditions may have led to increased failure to inhibit responses until the shape was correctly identified, which would be revealed by higher FA rates in double-feature than in single-feature conditions. FA rates, however, were similar in both conditions. Since no differences in FA rates were obtained for single- and double-feature conditions, the redundancy gain and race model violations obtained with double features cannot be blamed on selective failures to inhibit responses in this condition. Fast responses causing FAs increase the observed amount of fast responses on both sides of the inequality. Since this increase is stronger on the right side with two terms than on the left side with only one term, a race model might mistakenly be adopted (Eriksen, 1988), however. The conservative valuation of whether the race model is violated strengthens the conclusion that race model violation is real for the color-motion combination.

In sessions with color-frequency and motion-frequency, the single-feature RTs differ by about $70-100 \mathrm{~ms}$, with minor overlap of the $95 \%$ confidence intervals (Fig. 2). Since the race model requires overlapping RT distributions, this may reduce redundancy gain caused by statistical facilitation as described by the race model. So the low redundancy gain in these cases may be underestimated not only due to high FA rates. Importantly, the color-motion conjunction causes the largest redundancy gain in the identification task, providing evidence for coactivation, which is contrary to expectations, considering that these features are used as cardinal examples of processing in separate pathways.

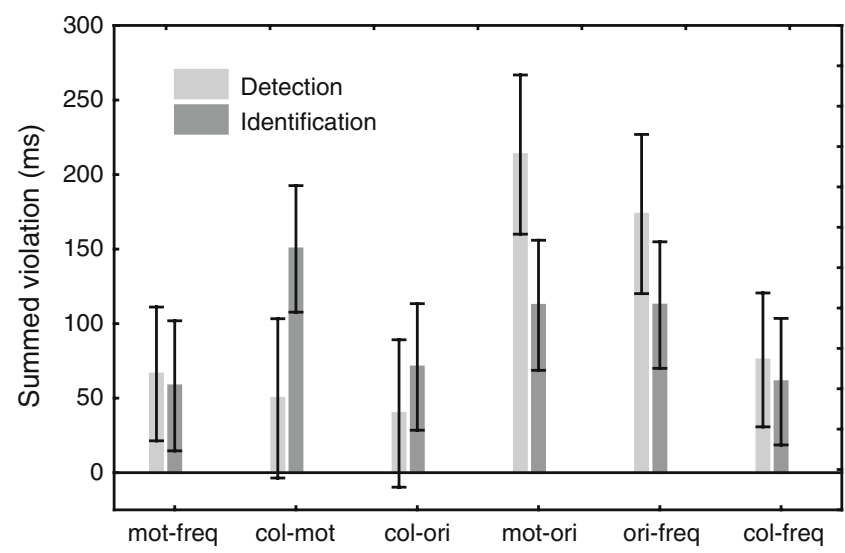

Fig. 4 Summed violationas described in the text for the different feature combinations in the identification task from Experiment 1 and the detection task from Poom (2009).The whiskers show $95 \%$ confidence intervals 
There is a possibility, however, that the different pattern of results might result from the different number of target elements. In the detection task, only one odd element had to be detected, whereas in the identification task, the target (and distractors) consisted of 11 elements. Maybe the limited color and motion signal in the detection task fail to coactivate, whereas the stronger signal conveyed by 11 elements do coactivate.

\section{Experiment 2}

In Experiment 2, using color and motion as shapedistinguishing features, the same S-shaped target was used as in Experiment 1 in both a detection and an identification task. Furthermore, Experiment 2 tested both tasks with a single group of observers, encouraged to withhold responses on no-go trials to minimize the FA rate, which was quite high in Experiment 1.

\section{Method}

Participants Sixteen participants (20-47 years old) with no color vision deficits were recruited to participate in the experiment. All had normal or corrected-to-normal vision. Informed consent was obtained from all the participants after the nature of the experiment was explained, and they were rewarded with cinema tickets for their participation.

Stimuli Only color, motion, and color-motion conjunctions were used here; otherwise, the stimulus parameters were the same as those described in Experiment 1. In the detection task, the S-shaped target was presented on go trials, and background noise elements alone were presented on no-go trials. The identification task was the same as in Experiment 1.

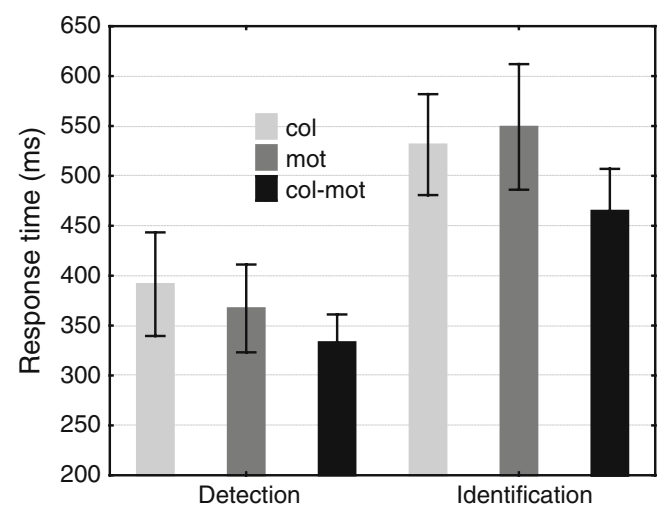

Fig. 5 Average response times across participants for color alone (col) and motion alone (mot) and for color and motion used in combination (col-mot) in the detection and identification task in Experiment 2. The whiskers show 95\% confidence intervals

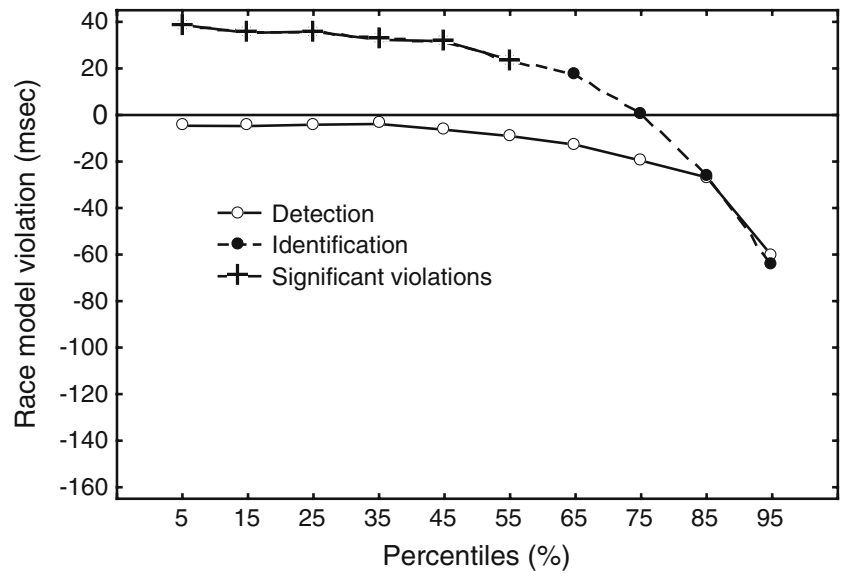

Fig. 6 Results from Experiment 2, using color and motion as shapedistinguishing features. Response time deviations from the race model boundary (line at zero) separately presented at each percentile demonstrate clear evidence for coactivation in the identification task (positive race model violations). The same features and shape failed to provide any evidence for coactivation in the detection task

Procedure The target occurred on $75 \%$ of the trials randomly interleaved with no-go trials. The detection and identification tasks were carried out in separate sessions, randomized between observers. Observers were encouraged to withhold responses on no-go trials to avoid FAs, and it was emphasized that FAs added extra trials.

\section{Results}

Figure 5 shows the mean RTs for single- and double-feature conditions in the detection and identification tasks. RTs from single-feature conditions overlap in both the detection and identification tasks, and the shortest RTs seem to have been obtained when the target was specified by both color and motion. Figure 6 shows that again, the identification task with color-motion conjunctions led to race model violations requiring coactivation of color and motion signals. In the detection task, however, color-motion conjunctions failed to provide any evidence for coactivation, which replicates the results obtained with a single-element target (Koene \& Zhaoping, 2007; Poom, 2009). A paired $t$-test showed that there was a significant difference between the two conditions shown in Fig. $6, t(9)=5.22, p<.001$. The proportion for the FA rate was reduced, as compared with Experiment 1; in the detection task, it was about $3 \%$, and in the identification task, it was $7 \%$.

\section{Discussion}

As in Experiment 1, coactivation was found in the identification task for the color-motion conjunction. The results from the detection task show no evidence for color- 
motion coactivation, even though the target was composed of the same number of elements and had the same shape as the target used in the identification task, replicating previous results obtained with single target elements (Koene \& Zhaoping, 2007; Poom, 2009). In the identification task, the target shape had to be identified and distinguished from distractor shapes embedded in noise elements, whereas in the detection task, the target just had to be detected and distinguished from trials containing only noise elements.

The FA rates from Experiment 1 were severely reduced from about $20 \%$ to $7 \%$ in the identification task and from $13 \%$ in the detection task with a single target element (Poom, 2009) to about 3\% using a target composed of multiple elements. This provides additional support that the results in the color-motion condition in Experiment 1 and the previous detection task were not influenced by high guessing rates. When FA rates are high, race model violations are conservative in providing evidence for coactivation, but, although the FA rates were reduced in the detection task, no race model violations were obtained.

\section{General discussion}

The segregation of color and motion is traditionally used as a cardinal example of feature-specific processing in separate pathways as exemplified by visual search tasks (Koene \& Zhaoping, 2007; Poom, 2009). In the identification task of Experiment 1, the greatest redundancy gains occurred for color-motion conjunctions, revealing clear evidence for coactivation. In Experiment 2, color-motion conjunctions failed to produce race model violations in the detection task, even though the same target was used as in the identification task, whereas again coactivation was found in the identification task.

Intuitively, one has to do two things to accomplish correct identification: First, detect and locate the target elements among the distractor elements, and thereafter, group the target elements to be able to identify the object. For example, in the feature integration theory, first, an early preattentive level of processing takes place, at which basic feature extraction occurs in parallel, and at a later stage, focused attention is required to conjoin the separate features into coherent objects (Treisman \& Gelade, 1980). So it is possible that detection, grouping, and identification are sequential processes within the same pathway or, alternatively, that separate routes of processing handle these tasks. In the first case, some kind of dependency between results from detection and identification is expected.

If features are separately processed at the pregrouping stages, there is still a possibility of coactivation at the postgrouping stages. If features coactivate at the pregrouping stage, no further gain may be possible at later stages, due to saturation. This may be an outline for a model explaining the opposite pattern of results in these two tasks. Color-motion conjunctions coactivate when the task is identification at a postgrouping stage, not when the task is detection at a pregrouping stage. Other feature combinations show larger redundancy gain in a detection task than in the identification task when measured by summed violations. These results indicate a dependency between processes.

In the sequential-processing view, detection is determined by activity in $\mathrm{V} 1$, where color and motion are separately processed, but other features activate common neural populations (Koene \& Zhaoping, 2007; Poom, 2009). In contrast, in area V2, there are cells tuned to all feature conjunctions, inclusive of color-motion conjunctions (Gegenfurtner, Kiper, \& Fenstemaker, 1996), which argues against V2 as a locus selectively engaged in the visual search detection task. Identification, on the other hand, is crucially dependent on object-selective neurons in the lateral occipital cortex (LOC) and inferior temporal (IT) cortex (Gulyas, Heywood, Popplewell, Roland, \& Cowey, 1994; Sary, Vogels, \& Orban, 1993; Self \& Zeki, 2004). One may conceptualize these brain areas as object maps of frequently encountered objects, as opposed to the location-specific saliency maps in cortical area V1. In identification tasks, race model violations can be modeled by coactivation on the object map, where objects specified by multiple features become more salient than single-feature objects. This process is comparable to coactivation on the saliency map, representing locations of salient features in the visual field.

In line with the object map model, object-selective neurons that respond regardless of whether the object is defined by motion, luminance, or texture size have been found in the temporal cortex (Sary et al., 1993). Self and Zeki (2004) found that motion-color defined objects activated LOC more than if either feature was used alone and found crossadaptation effects, which may indicate that this is a neural site for coactivation. Furthermore, these areas are known to receive mayor contributions from both the color-specialized parvocelluar and the motion-specialized magnocelluar pathways (Merigan \& Maunsell, 1993).

FA rates in identification are higher and RTs are longer than in the detection task, probably due to more top-down influences in higher processing stages. Accordingly, Nothdurft (2006) found longer RTs when the task was to identify the feature that made a target element pop out, rather than just detecting an odd element. This does not mean that detection tasks are free from top-down influences. In the widely recognized saliency map models, pop-out of odd targets occurs where bottom-up driven activity in corresponding loci at the saliency map reaches a specific threshold (Cave \& Wolfe, 1990; Koch \& Ullman, 1985; Müller, Heller, \& Ziegler, 1995; Wolfe, 1994, 2001). Still, attention may 
influence the weights assigned to different features. In pregrouping detection tasks, it is known that RTs are shorter when target-defining features repeat, rather than change, between trials (Found \& Müller, 1996). Krummenacher, Müller, and Heller (2001) found that, in detection tasks, when feature weights need to be shifted under variable-feature conditions, race model violations are less reliably obtained than under constant-feature conditions. Their explanation was that limited attentional weights are better balanced between features in redundant conditions, permitting their features to interact. Greater weight is assigned to only one feature at a time on feature-specific maps and persists to the next trial (Müller et al., 1995). If the target dimension is known in advance, signals from that dimension are amplified, and a time-consuming weight shift occurs when the feature changes between trials.

This is in line with Wolfe's (2001) Guided Search model, where both preattentional saliency-driven bottom-up and observer driven top-down guidance that directs attention toward target features is used to direct attention in visual search. When the target-defining feature is known, both processes act in concert to speed up detection. Analyses of ERPs obtained during weight shifts demonstrate the involvement of relatively early perceptual processes (Gramann, Toellner, Krummenacher, Eimer, \& Müller, 2007). Here, although conditions for coactivation were optimized by running blocks with constant-feature conditions, no race model violations were found in the pregrouping task for color-motion in Experiment 2 or in the previous study (Poom, 2009). Whether similar costs, caused by weight shifts between features, occur in postgrouping stages as in pregrouping stages is an interesting question for future research.

The pattern of results obtained by comparing RT redundancy gain in detection and identification may be a general effect of task demand. Kastner, Busse, and Treue (2006) measured RTs for identification of a particular color, direction of motion, or both combined in redundant conditions and found evidence for coactivation. On the other hand, detection of coherent motion is facilitated by texture and disparity signals, but not by color (Poom \& Börjesson, 2006). This may imply that detection tasks generally, not only those involving visual search, are mediated by separate color and motion processes and that identification generally, and not only shape or object identification, is mediated by processes where color and motion produce coactivation.

In summary, comparing the results for identification and detection suggests that color and motion signals are kept separate until identification processes take place from signals converging at the object map and, therefore, produce more redundancy gain than do other feature pairs that have converged at lower stages of processing. Thus, in visual search, the decision stage relies on signals on separate color- and motion-specific saliency maps. These separate activities therefore lead to statistical facilitation for color-motion conjunctions in visual search tasks, as described by the race model (Koene \& Zhaoping, 2007; Poom, 2009). In identification tasks, involving the temporal cortex, where motion and color signals are finally integrated (Self \& Zeki, 2004), the decision stage relies on coactivation of object-selective neurons on the object map, responding to specific objects irrespectively of whether they are defined by motion or color.

Author Note This research was supported by the Swedish Research Council and the Magnus Bergwall foundation.

\section{References}

Cave, K. R., \& Wolfe, J. M. (1990). Modeling the role of parallel processing in visual search. Cognitive Psychology, 22, 225-271.

Chambers, C. D., \& Brown, M. (2003). Timing accuracy under Microsoft Windows revealed through external chronometry. Behavior Research Methods, Instruments, \& Computers, 35, 96-108.

Colonius, H., \& Diederich, A. (2006). The race model inequality: Interpreting a geometric measure of the amount of violation. Psychological Review, 113, 148-154.

Diedrich, A., \& Colonius, H. (1987). Intersensory facilitation in the motor component? A reaction time analysis. Psychological Research, 49, 23-29.

Eriksen, C. W. (1988). A source of error in attempts to distinguish coactivation from separate activation in the perception of redundant targets. Perception \& Psychophysics, 44, 191-193.

Feintuch, U., \& Cohen, A. (2002). Visual attention and co-activation of response decisions for features from different dimensions. Psychological Science, 13, 362-370.

Field, D. J., Hayes, A., \& Hess, R. F. (1993). Contour integration by the human visual system: Evidence for a local "association field. Vision Research, 33, 173-193.

Found, A. P., \& Müller, H. J. (1996). Searching for feature targets on more than one dimension: Investigating the dimension weighting account. Perception \& Psychophysics, 58, 88-101.

Gegenfurtner, K. R., Kiper, D. C., \& Fenstemaker, S. B. (1996). Processing of color, form, and motion in macaque area V2. Visual Neuroscience, 13, 161-172.

Gramann, K., Toellner, T., Krummenacher, J., Eimer, M., \& Müller, H. J. (2007). Brain electrical correlates of dimensional weighting: An ERP study. Psychophysiology, 44, 277-292.

Gulyas, B., Heywood, C. A., Popplewell, D. A., Roland, P. E., \& Cowey, A. (1994). Visual form discrimination from color or motion cues: Functional-anatomy by positron emission tomography. Proceedings of the National Academy of Sciences, 91, 9965-9969.

Hughes, H. C., \& Townsend, J. T. (1998). Varieties of binocular interaction in human vision. Psychological Science, 9, 53-60.

Kastner, S., Busse, L., \& Treue, S. (2006). Feature-based attentional integration of color and visual motion. Journal of Vision, 6, 269284.

Koch, C., \& Ullman, S. (1985). Shifts in selective visual attention: Towards the underlying neural circuitry. Human Neurobiology, 4, 219-227. 
Koene, A. R., \& Zhaoping, L. (2007). Feature-specific interactions in saliency from combined feature contrasts: Evidence for a bottom-up saliency map in V1. Journal of Vision, 7(7, Art. 6), $1-14$.

Krummenacher, J., Müller, H. J., \& Heller, D. (2001). Visual search for dimensionally redundant pop-out targets: Evidence for parallel-coactive processing of dimensions. Perception \& Psychophysics, 63, 901-917.

Merigan, W. H., \& Maunsell, J. H. R. (1993). How parallel are the primate visual pathways? Annual Review of Neuroscience, 16, $369-402$.

Miller, J. (1982). Divided attention: Evidence for coactivation with redundant signals. Cognitive Psychology, 14, 247-279.

Mordcoff, J. T., Miller, J., \& Roch, A. C. (1996). Absence of coactivation in the motor component: Evidence from psychophysiological measures of target detection. Journal of Experimental Psychology: Human Perception \& Performance, 22, 2541.

Mordkoff, J. T., \& Yantis, S. (1993). Dividing attention between color and shape: Evidence of coactivation. Perception \& Psychophysics, 53, 357-366.

Müller, H. J., Heller, D., \& Ziegler, J. (1995). Visual search for singleton feature targets within and across feature dimensions. Perception\& Psychophysics, 57, 1-17.

Nothdurft, H. C. (1993). The role of features in preattentive vision: Comparison of orientation, motion and color cues. Vision Research, 33, 1937-1958.

Nothdurft, H. C. (2006). Salience and target selection in visual search. Visual Cognition, 14, 514-542.

Poom, L. (2001). Visual inter-attribute contour completion. Perception, 30, 855-865.

Poom, L. (2002). Visual binding of luminance, motion and disparity edges. Vision Research, 42, 2577-2591.

Poom, L. (2009). Integration of color, motion, orientation and spatial frequency in visual search. Perception, 38, 708-718.
Poom, L., \& Börjesson, E. (2006). Color, polarity, disparity, and texture contributions to the detection of global motion. Perception, 34, 1193-1203.

Raab, D. H. (1962). Statistical facilitation of simple reaction times. Transactions of the New York Academy of Sciences, 24, 574-590.

Sary, G., Vogels, R., \& Orban, G. A. (1993). Cue-invariant shape selectivity of macaque inferior temporal neurons. Science, 260, 995-997.

Segalowitz, S. J., \& Graves, R. E. (1990). Suitability of the IBM XT, $\mathrm{AT}$, and PS/2 keyboard, mouse, and game port as response devices in reaction time paradigms. Behavior Research Methods, Instruments, \& Computers, 22, 283-289.

Self, M. W., \& Zeki, S. (2004). The integration of color and motion by the human visual brain. Cerebral Cortex, 15, 1270-1279.

Todd, J. W. (1912). Reaction to multiple stimuli. Archives of Psychology, 25, 1-65.

Treisman, A., \& Gelade, G. (1980). A feature-integration theory of attention. Cognitive Psychology, 12, 97-136.

Ulrich, R., Miller, J., \& Schrötter, H. (2007). Testing the race model inequality: An algorithm and computer programs. Behavior Research Methods, 39, 291-302.

von Mühlenen, A., \& Müller, H. J. (2001). Visual search for motionform conjunctions: Is form discriminated within the motion system? Journal of Experimental Psychology: Human Perception and Performance, 27, 707-718.

Wolfe, J. M. (1994). Guided Search 2.0: A revised model of visual search. Psychonomic Bulletin \&Review, 1, 202-238.

Wolfe, J. M. (2001). Guided Search 4.0: A guided search model that does not require memory for rejected distractors [Abstract]. Journal of Vision, 1 (3, Art. 349).

Zehetleitner, M., Krummenacher, J., \& Müller, H. J. (2009). The detection of feature singletons defined in two dimensions is based on saliency summation, rather than on serial exhaustive or interactive race architectures. Attention, Perception, \& Psychophysics, 71, 1739-1759. 